\title{
Magnetosphere-ionosphere coupling during periods of extended high auroral activity: a case study
}

\author{
S. Liléo ${ }^{1}$, G. T. Marklund ${ }^{1}$, T. Karlsson ${ }^{1}$, T. Johansson ${ }^{1}{ }^{*}$, P.-A. Lindqvist ${ }^{1}$, A. Marchaudon $^{2}$, A. Fazakerley ${ }^{3}$, \\ C. Mouikis ${ }^{4}$, and L. M. Kistler ${ }^{4}$ \\ ${ }^{1}$ Space and Plasma Physics, School of Electrical Engineering, Royal Institute of Technology (KTH), Stockholm, Sweden \\ ${ }^{2}$ LPCE, CNRS-Université d'Orléans, Orléans, France \\ ${ }^{3}$ Mullard Space Science Laboratory, University College London, UK \\ ${ }^{4}$ Space Science Center, University of New Hampshire, Durham, USA \\ *now at: Laboratory for Atmospheric and Space Physics, University of Colorado, Boulder, CO, USA
}

Received: 30 June 2007 - Revised: 23 January 2008 - Accepted: 1 February 2008 - Published: 26 March 2008

\begin{abstract}
Results are presented from a case study of a plasma boundary crossing by the Cluster spacecraft during an extended period of high auroral activity. The boundary between the magnetotail lobe region of the Southern Hemisphere and the plasma sheet boundary layer, was characterized by intense electric and magnetic field variations, structured upward accelerated ion beams, narrow-scale large field-aligned Poynting fluxes directed upward away from the ionosphere, and a relatively sharp plasma density gradient.

The observations are shown to be consistent with the concept of a multi-layered boundary with temporal and/or spatial variations in the different layers. $\mathrm{H}^{+}$and $\mathrm{O}^{+}$ion beams are seen to be accelerated upwards both by means of a fieldaligned electric field and by magnetic pumping caused by large-amplitude and low-frequency electric field fluctuations. The peak energy of the ion beams may here be used as a diagnostic tool for the temporal evolution of the spatial structures, since the temporal changes occur on a time-scale shorter than the times-of-flight of the detected ion species.

The case study also shows the boundary region to be mainly characterized by a coupling of the detected potential structures to the low ionosphere during the extended period of high auroral activity, as indicated by the intense fieldaligned Poynting fluxes directed upward away from the ionosphere.
\end{abstract}

Keywords. Ionosphere (Auroral ionosphere; Electric fields and currents; Ionosphere-magnetosphere interactions)

$\overline{\text { Correspondence to: } \text { S. Liléo }}$

(sonia.lileo@ee.kth.se)

\section{Introduction}

The plasma sheet boundary layer (PSBL) is a rather dynamic transition region between the magnetotail lobes and the central plasma sheet (Eastman et al., 1984). It undergoes significant temporal variations on a time-scale of minutes (Figueiredo et al., 2005) and is of great importance in the transmission of energy flux between the distant tail and the auroral acceleration region (Keiling et al., 2000).

Large field-aligned Poynting fluxes dominantly directed towards the ionosphere and associated with Alfvén waves have been shown to occur in the PSBL region during the expansion phase of substorms (Keiling et al., 2000, 2005), and associated with intense auroral structures (Wygant et al., 2000, 2002; Keiling et al., 2002). Large field-aligned Poynting fluxes directed towards the ionosphere have also been detected in the PSBL coupled with quasi-static negative/positive potential structures associated with field-aligned currents flowing upward/downward and in magnetic conjunction with auroral/black auroral structures (Figueiredo et al., 2005; Johansson et al., 2004). Furthermore, the coexistence of quasi-static potential structures and travelling Alfvén waves may occur rather frequently in the PSBL (e.g. Karlsson et al., 2004; Johansson et al., 2004), cooperating efficiently for the transmission of energy between the distant magnetotail and the auroral ionosphere.

In a recent study by Hwang et al. (2006) it was shown that downgoing field-aligned Poynting fluxes from a high altitude source are mostly associated with curved auroral forms, whereas upgoing field-aligned Poynting fluxes from a low altitude source tend to be associated with straight auroral arcs (sheetlike events). The degree by which a potential structure is coupled to the low ionosphere was shown to depend

Published by Copernicus Publications on behalf of the European Geosciences Union. 
on the type of structure: sheetlike events appear to have a strong contribution from ionospheric fields and are therefore a strong coupling of the potential structures to the ionosphere (low closure degree), meaning that part of the potential structure maps to the ionosphere; curved events are generated by an high altitude source and are associated with potential structures decoupled from the ionosphere (high closure degree), i.e. the potential structures close above the ionosphere forming U-shaped structures.

In this paper we present and analyze electric field (Electric Field and Wave instrument, EFW, Gustafsson et al., 1997), magnetic field (Flux Gate Magnetometer, FGM, Balogh et al., 1997), electron (Plasma Electron And Current Experiment, PEACE, Johnstone et al., 1997) and ion (Cluster Ion Spectrometry, CIS, Rème et al., 1997) data for a Cluster crossing of the boundary between the magnetotail lobe region and the PSBL, that took place on 8 May 2003, in the Southern Hemisphere, at a geocentric distance of about 6 Earth radii. The event occurred during a period of highintensity long-duration continuous AE activity (HILDCAA). The concept of HILDCAA was introduced by Tsurutani and Gonzalez (1987) as periods characterized by intense activity in the auroral region that occur during high-speed solar wind streams. HILDCAA periods are defined as intervals where the auroral electrojet index (AE index) reaches, at least, $1000 \mathrm{nT}$; the AE values never drop below $200 \mathrm{nT}$ for more than $2 \mathrm{~h}$ at a time; the durations are greater than 2 days, and they occur outside the main phases of magnetic storms.

\section{Observations}

On 8 May 2003, between 17:40 and 18:00 UT, the Cluster spacecraft 1 crossed the evening MLT sector of the Southern Hemisphere auroral oval at an altitude of approximately 5 Earth radii. The spacecraft moved equatorward, crossing the boundary between a rather empty tail lobe region, that maps to the polar cap (PC), and the denser PSBL at approximately 17:47:30 UT. The AE index for this event indicates that it occurs during a period of high-intensity long-duration continuous AE activity (HILDCAA). Furthermore, the time of the event coincides with the peak (AE 1400 nT) of the expansion phase of a strong substorm, during a non-stormtime period. The velocity of the solar wind was high, 750 $\mathrm{km} / \mathrm{s}$ approximately, as measured by the ACE satellite. The interplanetary magnetic field pointed southward during the whole interval.

Figure 1 shows data measured by Cluster spacecraft 1 during this event.

The three first panels of Fig. 1 show the ion flux spectrograms versus pitch angle and time for the $\mathrm{H}^{+}$ions for the energy intervals $200-1000 \mathrm{eV}, 1-10 \mathrm{keV}$ and $10-38 \mathrm{keV}$, respectively. A pitch angle of $0^{\circ}$ corresponds to upgoing and $180^{\circ}$ to downgoing ions in the Southern Hemisphere. The ion flux spectrogram versus energy and time for the upgo- ing $\left(0-40^{\circ}\right.$ pitch angle) $\mathrm{H}^{+}$ions, is shown in panel 4 . The superposed black line indicates the corresponding characteristic energy. The next four panels show in a similar way the corresponding spectrograms for the $\mathrm{O}^{+}$ions.

The EFW experiment on board the Cluster spacecraft has four spherical sensors located at the ends of two long and orthogonal wire booms in the spin plane of the satellite (Gustafsson et al., 1997). The electric field is determined by measuring the potential difference between two pairs of opposing probes (p12 and p34). However, probe 1 on spacecraft 1 is out of function during the date of the event here presented. The output signals from probes 2 and 3 (p2 and p3, sampled at $5 \mathrm{~s}^{-1}$ ) were used to calculate p32 instead, which together with p34 (sampled at $25 \mathrm{~s}^{-1}$ and averaged to match the lower sampling rate of $\mathrm{p} 2$ and $\mathrm{p} 3$ ) allow us to determine the two components of the electric field in the spin plane. The electric field component along the spin axis is not measured but can be reconstructed using the assumption $\boldsymbol{E} \times \boldsymbol{B}=0$. This is a fairly safe assumption, considering that the altitude of the Cluster spacecraft is likely to be well above the acceleration region. The FGM experiment provides measurements of the local magnetic field. In order to extract the background geomagnetic field from the measured magnetic field, a polynomial function was fitted to a large-time window $(\sim 4 \mathrm{~h})$ of measured magnetic field data. The residual magnetic field was then computed by subtracting the fitted large-scale background magnetic field. Minimum variance analysis (MVA) was then applied to the electric field data and to the residual magnetic field data, in each of the four subregions separated by the red vertical lines plotted in Fig. 1, in order to determine the direction along which the electric field and the magnetic field better minimize and maximize, respectively, in each of the subregions. The results obtained from applying MVA to the electric field are rather consistent with the results from the MVA of the magnetic field, showing however less variance. Therefore, the orientation determined from the MVA of the electric field for each of the defined subregions, is here considered to best represent the alignment of the current sheet traversed by the spacecraft in each subregion. A coordinate system $\left(\boldsymbol{e}_{B}, \boldsymbol{e}_{t}, \boldsymbol{e}_{n}\right)$ was then defined, where $\boldsymbol{e}_{B}$ is parallel to the background magnetic field direction, $\boldsymbol{e}_{t}$ is tangential to the current sheet pointing roughly eastwards, and $\boldsymbol{e}_{n}$ is normal to the current sheet pointing towards lower latitudes. The normal component of the electric field ( $\left.E_{\text {normal }}\right)$, mapped to the ionosphere, is plotted in the last panel of Fig. 1. The tangential component is relatively small during all the time interval presented and is therefore not presented. The reason for mapping the electric field data to the ionosphere is to facilitate the comparison of the data here presented with data measured at different altitudes reported in other studies. The ionospheric level is therefore chosen only as a reference level.

The boundary between the magnetotail lobe region and the PSBL (17:47:30-17:51 UT) was divided into four small scale regions separated by the red vertical lines shown in Fig. 1. 


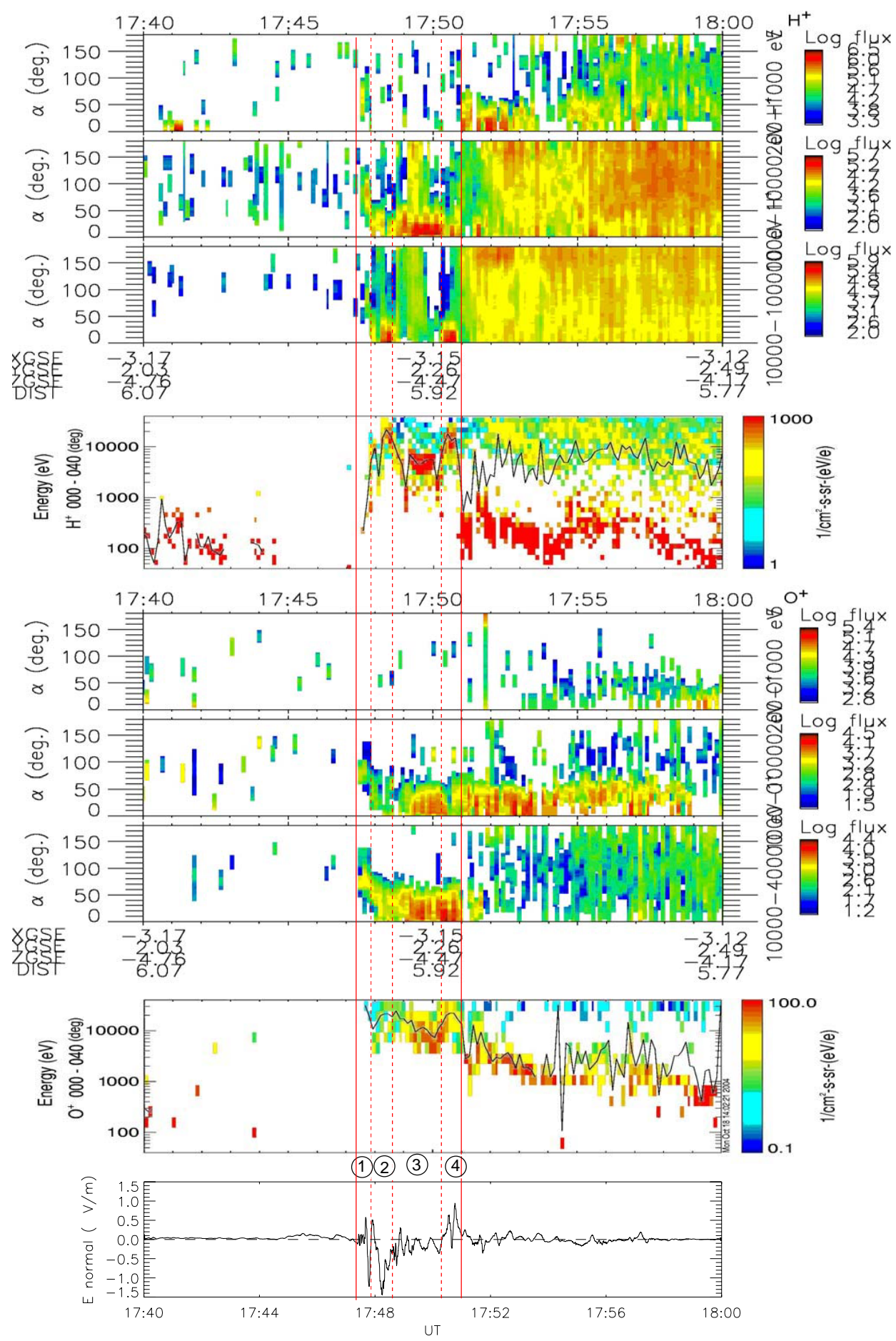

Fig. 1. CIS ion data measured on board Cluster spacecraft 1 on 8 May 2003. Panels 1 to $3-\mathrm{H}^{+}$ion flux spectrogram versus pitch angle and time for the energy intervals $200-1000 \mathrm{eV}, 1-10 \mathrm{keV}$ and $10-38 \mathrm{keV}$, respectively. Pitch angle of $0^{\circ}$ corresponds to upgoing and $180^{\circ}$ to downgoing ions on the Southern Hemisphere. Panel 4 - Ion flux spectrogram versus energy and time for the upgoing (0-40 pitch angle) $\mathrm{H}^{+}$ions. The superposed black line indicates the corresponding characteristic energy. Panels 5 to $7-\mathrm{O}^{+}$ion flux spectrogram versus pitch angle and time for the energy intervals $200-1000 \mathrm{eV}, 1-10 \mathrm{keV}$ and $10-38 \mathrm{keV}$, respectively. Panel 8 - Ion flux spectrogram versus energy and time for the upgoing $\left(0-40^{\circ}\right.$ pitch angle $) \mathrm{O}^{+}$ions. The superposed black line indicates the corresponding characteristic energy. Panel 9 - Normal component of the electric field mapped to the ionosphere. 
Moving from the tail lobe region into the PSBL, the Cluster spacecraft 1 encounters at 17:47:30 a narrow region (region 1) characterized by an asymmetric convergent electric field structure (associated with a negative S-shaped potential structure) with mapped peak intensity of $\sim 1 \mathrm{~V} / \mathrm{m}$. A signature of an $\mathrm{O}^{+}$ion conic with a pitch angle between 50 and 100 degrees (panels 6 and 7), and possibly also of an $\mathrm{H}^{+}$ion conic (panels 1 to 3 ), are observed in this region. Region 2 is characterized by upflowing $\mathrm{H}^{+}$and $\mathrm{O}^{+}$ion beams with peak energies of about $20 \mathrm{keV}$. An asymmetric convergent electric field structure (associated with a negative S-shaped potential structure) is crossed in this region, with mapped peak intensity of $\sim 1.5 \mathrm{~V} / \mathrm{m}$. Region 3 is relatively broader and shows electric field fluctuations with amplitudes of about $200 \mathrm{mV} / \mathrm{m}$ and frequency of $\sim 30 \mathrm{mHz}$. Upflowing $\mathrm{H}^{+}$and $\mathrm{O}^{+}$ion beams with peak energies of 5 and $10 \mathrm{keV}$, respectively, are detected in this region. Region 4 shows a mainly unipolar electric field structure with peak intensity of about $1 \mathrm{~V} / \mathrm{m}$ associated with upflowing $\mathrm{H}^{+}$and $\mathrm{O}^{+}$ion beams with peak energies of 15 and $20 \mathrm{keV}$, respectively.

For times later than 17:51 UT one may distinguish two different ion populations, most likely the hot plasma sheet population (green and yellow in flux, panel 4, and blue and green in flux, panel 8) flowing fairly isotropic, and an upgoing cold ionospheric population (red in flux panels 4 and 8). The upgoing ionospheric population has a maximum energy in the time interval between 17:47:30 and 17:51 UT, as a result of the acceleration processes below the spacecraft. Due to the rather small electric field fluctuations observed for times later than 17:51 UT, a further analysis of these data will not be discussed here.

Data from the PEACE and the FGM experiments onboard the Cluster spacecraft 1 measured during this event are presented in Fig. 2.

In the top three panels are shown energy flux spectrograms versus energy and time for electrons at pitch angles 0,90 and $180^{\circ}$, respectively. A pitch angle of $0^{\circ}$ corresponds to upgoing electrons and $180^{\circ}$ to downgoing electrons, as the spacecraft is crossing the Southern Hemisphere. The potential calculated from the integration of the measured electric field along the orbit is shown in the fourth panel. The normal component of the electric field ( $\left.E_{\text {normal }}\right)$, is shown in the fifth panel with black line; The green line represents the tangential component of the residual magnetic field, $\left(B_{\text {tangential }}\right)$. Panel 6 shows the field-aligned current density distribution (FAC) calculated from the residual magnetic field and assuming an infinite current sheet, stationary in space. Colored with blue are the regions of upward current and with red the downward current regions. The last panel shows the fieldaligned component of the Poynting flux vector, with positive values indicating upward field-aligned flux. The Poynting flux vector was estimated from the 3-dimensional electric and magnetic field vectors, after the electric field samples have been averaged to match the lower sampling rate of the magnetic field. The electric and magnetic field values, as well as the FAC and the Poynting flux, were then mapped down to the ionosphere by assuming that the magnetic field is a dipole field and the magnetic field lines are equipotentials, and assuming no current sources and no power dissipation as well. All values plotted in Fig. 2 are mapped values. The mapping to the ionosphere was done only to simplify comparisons as referred above. The mapped values should not be considered as being the real ionospheric values. The red vertical lines define once more the subregions into which the boundary between the tail lobe region and the PSBL was divided.

A sharp boundary between the magnetotail lobe region and the PSBL can be identified in the electron flux spectrograms in Fig. 2 (panels 1 to 3) at around 17:47:30 UT. The region crossed by Cluster spacecraft 1 between 17:47:30 and 17:51 UT (indicated by two solid red vertical lines) is populated mainly by hot electrons with energies greater than $4 \mathrm{keV}$. The estimated perpendicular potential shows a relatively small potential drop associated with region 1 , and net potential differences of about 6 and $3 \mathrm{kV}$ associated with the regions 2 and 4, respectively. In order to facilitate the analysis of the electric field normal component and of the magnetic field tangential component, a zoom plot for the time interval 17:47-17:52 UT is presented in Fig. 3. The electric field normal component shows intense fluctuations in the entire boundary region. Mapped peak amplitudes of about $1.5 \mathrm{~V} / \mathrm{m}$ and $1 \mathrm{~V} / \mathrm{m}$ are measured in regions 2 and 4 , respectively. In the intermediate region 3 , the electric field normal component oscillates with a frequency of about $30 \mathrm{mHz}$. Large field-aligned currents with mapped densities up to $30 \mu \mathrm{A} / \mathrm{m}^{2}$ are measured in the region in concern (next to last panel, Fig. 2). A pair of downward and upward FACs is associated with the density gradient between the tail lobe region and the PSBL (region 1). A weakening of the field-aligned current density is detected in region 2 , whereas a mainly upward directed field-aligned current is detected in regions 3 and 4. The estimate of the perpendicular potential in region 3 is most likely invalid due to the temporal nature of the electric field fluctuations in this region. The estimated field-aligned Poynting flux plotted in the last panel of Fig. 2 shows intense field-aligned Poynting flux directed upward away from the ionosphere in particularly in regions 1 and 2, and also in region 4 (although much weaker), reflecting the anti-correlation between the electric and the magnetic field components seen in these regions (Fig. 3).

Due to the large time separation between the Cluster spacecraft (about $1 \mathrm{~h}$ ), significant modifications of the plasma sheet occur between the passages, not allowing a clear identification of the plasma sheet boundary, neither of the electric field structures in concern, for the other Cluster passages. Thus, only data from Cluster spacecraft 1 is presented. 


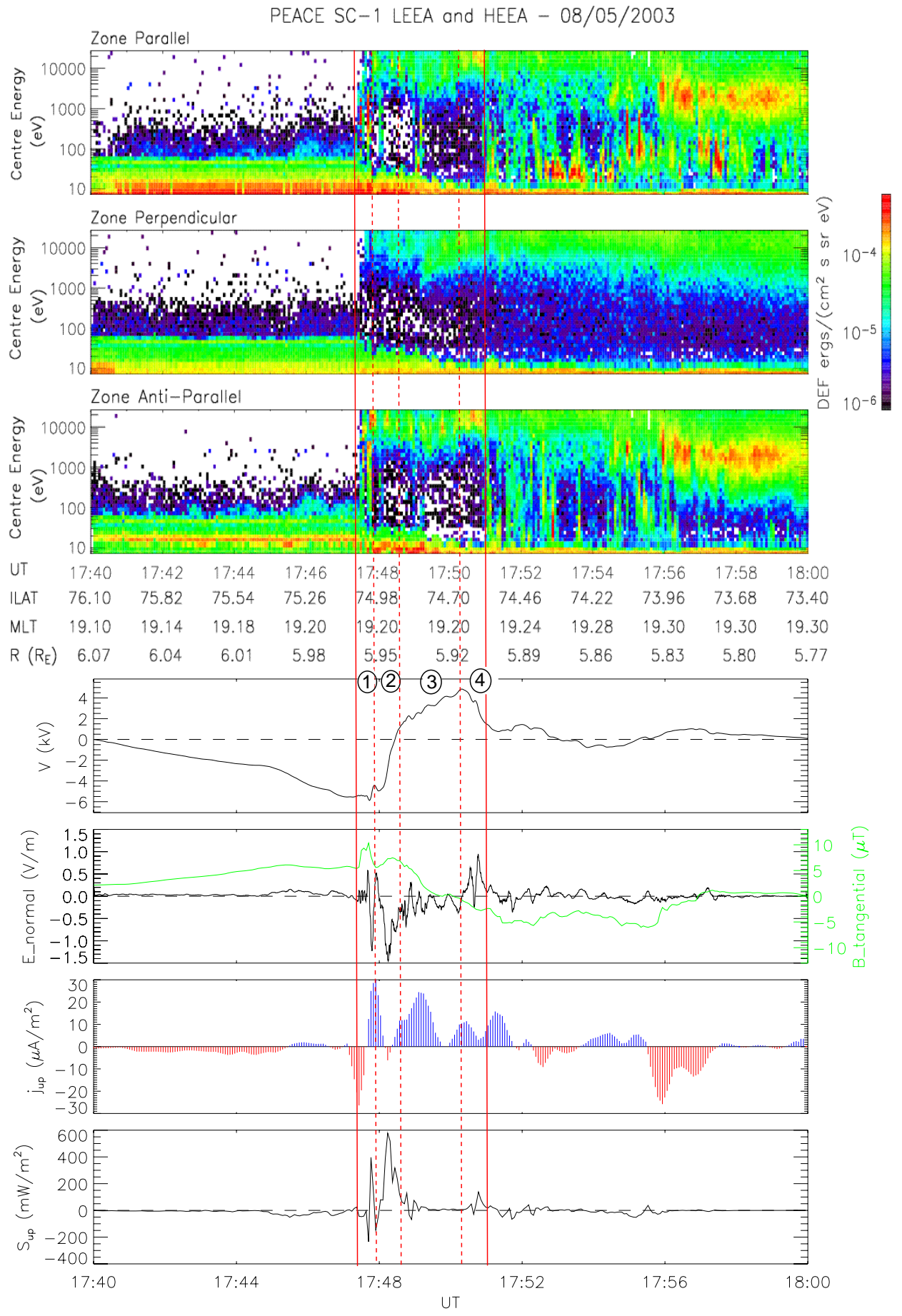

Fig. 2. Data from Cluster spacecraft 1 for the time interval 17:40 and 18:00 UT on 8 May 2003. Panels 1 to 3 - Electron energy flux spectrogram versus energy and time at pitch angles 0,90 and $180^{\circ}$, respectively. Pitch angle of $0^{\circ}$ corresponds to upgoing and $180^{\circ}$ to downgoing electrons on the Southern Hemisphere. Panel 4 - Calculated perpendicular potential. Panel 5 - Normal component of the electric field (black line); Tangential component of the magnetic field (green line). Panel 6 - Field-aligned current density distribution, with blue color showing upflowing currents and red color downflowing currents. Panel 7 - Field-aligned component of the Poynting flux vector, with positive values indicating upward field-aligned flux. All quantities are mapped to the ionosphere. 


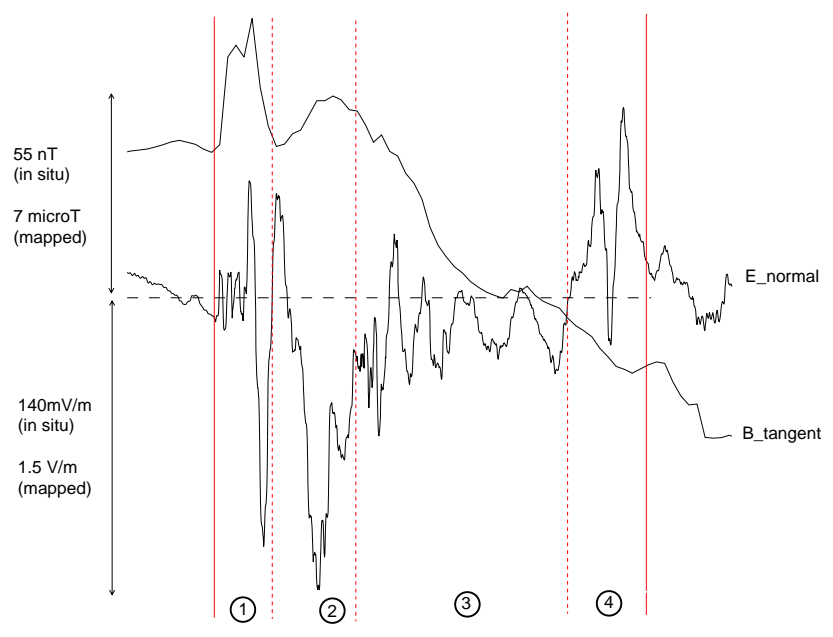

Fig. 3. Zoom plot of the electric field normal component and of the magnetic field tangent component for the time interval 17:4717:52 UT. The four regions in analysis are delimited by the red vertical lines.

\section{Discussion}

We present Cluster measurements for an auroral crossing of the Southern Hemisphere evening sector at an altitude of about $5 R_{E}$ during a period of extended high auroral activity (HILDCAA period). The spacecraft crossed the tail lobe boundary at 17:47:30 UT entering a rather structured PSBL. Our analysis focuses on the region of the PSBL right adjacent to the tail lobe boundary, crossed by the Cluster spacecraft 1 between 17:47:30 and 17:51 UT. This region is about $62 \mathrm{~km}$ broad, when mapped to the ionosphere, and is characterized by an almost omnidirectional electron population with energies above $4 \mathrm{keV}$, and rather low electron flux density for energies below. A higher flux of $\mathrm{H}^{+}$compared to $\mathrm{O}^{+}$is observed through all the event, as shown to occur for substorms during non-storm-time periods by Korth et al. (2002). Upflowing $\mathrm{H}^{+}$and $\mathrm{O}^{+}$ions accelerated to energies of $20 \mathrm{keV}$ are detected in the region between 17:47:30 and 17:51 UT, associated with intense electric and magnetic field fluctuations.

It is shown that four distinct regions of either temporal or spatial field variations (named by regions 1 to 4, Figs. 1, 2 and 3) may be distinguished. The main characteristics of each of these regions are summarized below.

Region 1. Narrow region characterized by an intense electric field (up to $1.2 \mathrm{~V} / \mathrm{m}$ mapped intensity) associated with a negative Sshaped potential structure and with $\mathrm{O}^{+}$and $\mathrm{H}^{+}$ion conics. A pair of downward and upward FACs is detected together with a rather strong peak ( $400 \mathrm{~mW} / \mathrm{m}^{2}$ mapped density) on the magnitude of the field-aligned Poynting flux component, directed upward away from the ionosphere.

Region 2. Intense electric field peak (1.5 V/m mapped) associated with a negative $\mathrm{S}$-shaped potential structure. The estimated perpendicular potential drop is about $6 \mathrm{kV}$. An upward flowing $\mathrm{H}^{+}$ion beam with a peak energy of $20 \mathrm{keV}$ is measured. Upward accelerated $\mathrm{O}^{+}$ions are also detected with relatively less flux density but similar peak energy. Strong field-aligned Poynting flux directed upward away from the ionosphere is also measured, associated with a weakening of the field-aligned current density.

Region 3. Electric field temporal fluctuations with peak-to-peak amplitude of approximately $300 \mathrm{mV} / \mathrm{m}$ (mapped to the ionosphere) and frequency of about $30 \mathrm{mHz}$ are detected associated with upward flowing $\mathrm{H}^{+}$and $\mathrm{O}^{+}$ion beams with characteristic energies of 5 and $10 \mathrm{keV}$, respectively. A region of mainly upflowing field-aligned currents is detected.

Region 4. Unipolar electric field structure associated with upward accelerated $\mathrm{H}^{+}$and $\mathrm{O}^{+}$ion beams, and a negative $\mathrm{S}$-shaped potential structure. The upflowing $\mathrm{H}^{+}$and $\mathrm{O}^{+}$ions have peak energies of 15 and $20 \mathrm{keV}$, respectively, and the estimated perpendicular potential drop is about $3 \mathrm{kV}$. A region of mainly upflowing field-aligned currents is detected, together with a small peak on the magnitude of the field-aligned Poynting flux component, directed upward away from the ionosphere.

A mismatch exists between the estimated perpendicular potential of the spatial structures crossed by spacecraft 1 in regions 2 and 4, and the peak energies of the $\mathrm{H}^{+}$and $\mathrm{O}^{+}$ ion beams measured in these regions. Note that for an Sshaped potential structure, the estimated perpendicular potential drop represents a maximum value of the corresponding parallel potential drop. The mismatch questions the validity of the hypothesis of negative potential structures traversed by the spacecraft in the regions 2 and 4 being responsible for the upward acceleration of the ions below the spacecraft. However, temporal changes of the potential structures may occur and must be taken into account, as well as the different times-of-flight of the $\mathrm{H}^{+}$and of the $\mathrm{O}^{+}$ions. An $\mathrm{H}^{+}$ ion with energy between 15 and $20 \mathrm{keV}$ takes approximately $20 \mathrm{~s}$ to travel between the acceleration region (supposed to be located at $6000 \mathrm{~km}$ altitude) and the location of the spacecraft. An $\mathrm{O}^{+}$ion within the same energy range takes four times longer to travel the same distance. This means that the detected $\mathrm{H}^{+}$ion beam in region 2 got its energy about $20 \mathrm{~s}$ prior to its detection, and the detected $\mathrm{O}^{+}$ion beam about $80 \mathrm{~s}$. During these time intervals, the parallel potential drops of the potential structures responsible for the acceleration of the ions appear to have decreased in strength from about $20 \mathrm{kV}$ to $\sim 6 \mathrm{kV}$ in region 2 , and from 20 to $\sim 3 \mathrm{kV}$ in region 4 .

Images from the Syowa All-Sky Imager (Syowa ASI) taken during the time interval 17:44-17:53 UT, show rapid and substantial auroral movements on a time-scale of $10 \mathrm{~s}$. The Syowa ASI is located in the Southern Hemisphere at 18:00 MLT and 67 degrees ILAT, thus not in conjugate position with the Cluster spacecraft during the event here described (19.2 MLT, 74.8 degrees ILAT). However, the analysis of the Syowa all-sky images of the area in vicinity of the magnetic footpoint of this event, gives an idea of the auroral dynamics during the time period in focus. Temporal 
variations of the auroral potential structures traversed by the Cluster spacecraft, on time scales shorter than the times-offlight of the $\mathrm{O}^{+}$and $\mathrm{H}^{+}$ions, are believed to contribute to the mismatch observed between the measured peak energy of the $\mathrm{H}^{+}$and $\mathrm{O}^{+}$ion beams and the estimated perpendicular potential drop. This mismatch may, in this case, be used to infer the time evolution of the spatial potential structures observed by the spacecraft.

Large-amplitude electric field fluctuations with a frequency of about $30 \mathrm{mHz}$ are detected by Cluster spacecraft 1 when passing through the region 3 . The relatively large ratio between the peak energies of the $\mathrm{O}^{+}$and of the $\mathrm{H}^{+}$upflowing ions observed in this region (ratio of about 2), suggests a mass-dependent acceleration process responsible for the ion energization. Magnetic moment pumping due to the large-amplitude and low-frequency electric field fluctuations detected, is a possible mechanism responsible for the generation of the $\mathrm{O}^{+}$and the $\mathrm{H}^{+}$ion beams detected in this region. The ratio of approximately 2 between the $\mathrm{O}^{+}$and the $\mathrm{H}^{+}$peak energies is in accordance with the results by Lundin and Hultqvist (1989). From the calculation of the $\mathrm{O}^{+} / \mathrm{H}^{+}$energy peak ratio of 46 upward flowing ion beams detected by the Viking satellite, an average value of 2.31 and a median of 1.57 was obtained by the authors. The authors concluded that a bimodal acceleration process consisting of a magnetic moment pumping mechanism caused by low-frequency transverse electric field fluctuations, together with a field-aligned quasi-static acceleration process, may generate the detected upward flowing ion beams. The accordance of the $\mathrm{O}^{+} / \mathrm{H}^{+}$ energy peak ratio in region 3 with the results by Lundin and Hultqvist (1989) confirms the mechanism of magnetic moment pumping as a probable process responsible for the ion energization in region 3 , and supports our assumption of a temporal nature of the electric field fluctuations in this region.

Large field-aligned Poynting flux directed upward away from the ionosphere with mapped density up to $600 \mathrm{~mW} / \mathrm{m}^{2}$, is measured in the regions 1 and 2 associated with the intense electric field peaks detected. A relatively weaker upward directed field-aligned Poynting flux is also measured associated with the electric field peak detected in region 4.

The physical meaning of the Poynting flux vector is expressed through the energy equation for the electromagnetic fields,

$\boldsymbol{E} \cdot \boldsymbol{j}=-\frac{\partial u}{\partial t}-\nabla \cdot \boldsymbol{S}$

with $u$ being the energy density and $S$ the energy flux of the electromagnetic field. The scalar product between the electric field $\boldsymbol{E}$ and the current $\boldsymbol{j}$ represents the loss of energy per unit time and per unit volume by the field. Assuming that the temporal derivative of the energy density is negligible (which is a reasonable assumption for quasistatic structures), it turns out that the change of energy by the field is given by the divergence of the Poynting flux vec- tor: negative/positive values of the divergence of $\boldsymbol{S}$ reflect loss/gain of energy by the field, which implies the presence of a sink/source of energy.

The observations here reported of large upward directed field-aligned Poynting fluxes measured by the Cluster spacecraft at an altitude of about 5 Earth radii imply a large positive derivative of the field-aligned component of the Poynting flux vector with increasing field-aligned distance from the Earth's surface, since the Poynting flux at the ionospheric footpoint of the magnetic field lines traversed by the Cluster spacecraft is expected to be much weaker than that measured by the Cluster spacecraft at high altitudes. Consequently, an energy source contributing for the gain of energy by the field may be present in the ionosphere below the spacecraft. However, since no measurements are available from the source region, nothing may be said about the derivatives of the normal and tangential components (referring to the coordinate system defined in the Observations section) of the Poynting flux vector along the respective directions over the source region. Therefore, a complete evaluation of the divergence of the Poynting flux vector is not possible for this case. However, allowing us to further develop our discussion based on the assumption that the change of the field-aligned Poynting flux component along the parallel direction is more significant than the two other terms of the divergence of $S$, one may conclude that the transport of energy from a possible source located below the spacecraft occurs mainly in the upward field-aligned direction. If so, which is the generation mechanism associated with this source of energy?

From a statistical study of the return current region performed by Hwang et al. (2006) using data from the FAST satellite, it was shown that the detected perpendicular electric field events having a significant contribution by ionospheric fields (S-shaped potential structures coupled to the ionosphere) were mainly associated with upgoing field-aligned Poynting fluxes, whereas decoupled potential structures (Ushaped closed potential structures) showed downgoing fieldaligned Poynting fluxes.

Several other studies have also discussed the observation of upward directed field-aligned Poynting flux above the ionosphere. Mishin et al. (2003) investigated electromagnetic and plasma density fluctuations within subauroral polarization streams. They concluded that electromagnetic energy in form of upward propagating Poynting flux should be observed above the ionosphere as a consequence of the mapping of potential distributions modified by plasma density (or correspondingly conductivity) variations to the magnetosphere. Lysak and Song (2002) and Streltsov and Lotko (2003) modelled the development of small-scale current structures in the auroral ionosphere associated with narrow-scale auroral arcs, based on the ionospheric feedback instability. It was shown that the ionosphere plays an important role for the formation and evolution of perpendicular electric field structures detected at high-altitudes, and therefore ionospheric effects should be taken into account when 
investigating high-altitude electric fields. The ionospheric feedback instability was interpreted as resulting from an enhancement of the ionospheric conductivity produced by the precipitation of electrons carried by the upward current region. When associated with a large-scale convection electric field, this conductivity enhancement can generate secondary field-aligned currents that further modify the ionospheric conductivity. The secondary field-aligned currents are carried by upward propagating Alfvén waves. When these are reflected back to the ionosphere they may generate a positive feedback, increasing the conductivity and allowing the instability to grow further. The free energy carried by the upward propagating Alfvén waves (in the form of upward field-aligned Poynting flux) results from a local reduction of the Joule heating (Lysak and Song, 2002).

In the event here investigated, the observed upward directed field-aligned Poynting fluxes in regions 2, 3, and 4 are interpreted as an indicator of the coupling of electric fields of ionospheric origin to Cluster altitudes. We speculate that the generation of these ionospheric electric fields is most probably associated with gradients in the ionospheric conductivity due to the precipitation of electrons in the upward current region. In order to allow current closure, an electric field is generated at the conductivity gradient at the ionospheric end of the current channel, modifying the potential distribution and mapping up to the magnetosphere. Consequent field-aligned currents will then distribute space charge along magnetic field lines in order to adapt them into the new equipotentials (Kan and Sun, 1985). As predicted by Mishin et al. (2003) upward flowing field-aligned Poynting flux should then be observed above the ionosphere.

\section{Conclusions}

We have presented intense electric and magnetic field variations measured at the boundary between the magnetotail lobe region and the PSBL during a period of extended high auroral activity and high solar wind speed, so called, HILDCAA period. The event analyzed also coincides with the peak of the expansion phase of a strong substorm. These conditions imply high solar wind energy, and an efficient energy transfer from the solar wind to the magnetosphere and to the ionosphere, suggested by the high AE index values measured during an extended period including the event. Furthermore, significant and rapid auroral movements (on a time-scale of at least $10 \mathrm{~s}$ ) are shown by all-sky images to occur in the surrounding area. The PSBL is known to be a rather dynamic region establishing the transition between open magnetic field lines of the magnetotail lobes and closed field lines of the central plasma sheet (Eastman et al., 1984), and hosting both travelling Alfvén waves and static FACs (Keiling et al., 2000; Johansson et al., 2004; Karlsson et al., 2004; Figueiredo et al., 2005).
The event scenario reported on here includes one potential structure collocated with the boundary between the tail lobe and the PSBL, and an other potential structure associated with a plasma boundary and a density gradient within the PSBL. In between these spatial potential structures there are large-amplitude electric field fluctuations with a frequency of about $30 \mathrm{mHz}$, accelerating the $\mathrm{H}^{+}$and $\mathrm{O}^{+}$ions upward, most probably by means of magnetic moment pumping. The comparison between the perpendicular potential drop estimated from the measured electric field and the peak energies of the upward accelerated $\mathrm{H}^{+}$and $\mathrm{O}^{+}$ion beams, allowed us to follow the time evolution of the spatial potential structures crossed by the spacecraft, since the times-of-arrival of the $\mathrm{O}^{+}$and $\mathrm{H}^{+}$ions are larger than the time-scale of the temporal variations of the potential structures.

Large field-aligned Poynting fluxes directed upward away from the ionosphere are detected and are interpreted as a consequence of the traversed potential structures being generated partly in the the low ionosphere. Whether such a coupling is favored during extended periods of high solar wind speed and efficient magnetosphere-ionosphere energy transfer (HILDCAA periods) seems probable since the ionosphere has in this case sufficiently long time to respond to the enhanced energy inputs. This is a matter of further investigation.

Acknowledgements. S. Liléo acknowledges the support of the Fundação para a Ciência e a Tecnologia (FCT) under the grant SFRH/BD/6211/2001. The AE index was obtained through the website of the World Data Center for Geomagnetism, Kyoto. ACE spacecraft data was obtained through the website of The ACE Science Center.

Topical Editor M. Pinnock thanks one anonymous referee for her/his help in evaluating this paper.

\section{References}

Balogh, A., Dunlop, M. W., Cowley, S. W., Southwood, D. J., Thomlinson, J. G., Glassmeier, K. H., Musmann, G., Lühr, H., Buchert, S., Acuña, M. H., Fairfield, D. H., Slavin, J. A., Riedler, W., Schwingenschuh, K., and Kivelson, M. G.: The cluster magnetic field investigation, Space Sci. Rev., 79, 65-91, 1997.

Eastman, T. E., Frank, L. A., Peterson, W. K., and Lennartsson, W.: The Plasma Sheet Boundary Layer, J. Geophys. Res., 89, 15531572, 1984.

Figueiredo, S., Marklund, G. T., Karlsson, T., Johansson, T., Ebihara, Y., Ejiri, M., Ivchenko, N., Lindqvist, P.-A., Nilsson, H., and Fazakerley, A.: Temporal and spatial evolution of discrete auroral arcs as seen by Cluster, Ann. Geophys., 23, 2531-2557, 2005 , http://www.ann-geophys.net/23/2531/2005/.

Gustafsson, G., Boström, R., Holback, B., Holmgren, G., Lundgren, A., Stasiewicz, K., Åhlén, L., Mozer, F. S., Pankow, D., Harvey, P., Berg, P., Ulrich, R., Pedersen, A., Schmidt, R., Butler, A., Fransen, A. W. C., Klinge, D., Fälthammar, C.-G., Lindqvist, P.-A., Christenson, S., Holtet, J., Lybekk, B., Sten, T. A., Tanskanen, P., Lappalainen, K., and Wygant, J.: The Electric Field 
and Wave experiment for the Cluster mission, Space Sci. Rev., 79, 137-156, 1997.

Hwang, K.-J., Lynch, K. A., Carlson, C. W., Bonnell, J. W., and Peria, W. J.: Fast Auroral Snapshot observations of perpendicular DC electric field structures in downward current regions: Implications, J. Geophys. Res., 111, A09206, doi:10.1029/2005JA011472, 2006.

Johansson, T., Figueiredo, S., Karlsson, T., Marklund, G., Fazakerley, A., Buchert, S., Lindqvist, P.-A., and Nilsson, H.: Intense high-altitude auroral electric fields - temporal and spatial characteristics, Ann. Geophys., 22, 2485-2495, 2004, http://www.ann-geophys.net/22/2485/2004/.

Johnstone, A. D., Alsop, C., Burge, S., Carter, P. J., Coates, A. J., Coker, A. J., Fazakerley, A. N., Grande, M., Gowen, R. A., Gurgiolo, C., Hancock, B. K., Narheim, B., Preece, A., Sheathier, P. H., Winningham, J. D., and Woodliffe, R. D.: PEACE: A Plasma Electron And Current Experiment, Space Sci. Rev., 79, 351-398, 1997.

Kan, J. R. and Sun, W.: Simulation of the westward travelling surge and Pi2 pulsations during substorms, J. Geophys. Res., 90, 10911-10 922, 1985.

Karlsson, T., Marklund, G. T., Figueiredo, S., Johansson, T., and Buchert, S.: Separating spatial and temporal variations in auroral electric and magnetic fields by Cluster multipoint measurements, Ann. Geophys., 22, 2463-2472, 2004, http://www.ann-geophys.net/22/2463/2004/.

Keiling, A., Wygant, J. R., Catell, C., Temerin, M., Mozer, F. S., Kletzing, C. A., Scudder, J., Russell, C. T., Lotko, W., and Streltsov, A. V.: Large Alfén wave power in the plasma sheet boundary layer during the expansion phase of substorms, Geophys. Res. Lett., 27, 3169-3172, 2000.

Keiling, A., Wygant, J. R., Cattell, C., Peria, W., Parks, G., Temerin, M., Mozer, F. S., Russell, C. T., and Kletzing, C. A.: Correlation of Alfvén wave Poynting flux in the plasma sheet at 4 $7 R_{E}$ with ionospheric electron energy flux, J. Geophys. Res., 107(A7), 1132, doi:10.1029/2001JA900140, 2002.

Keiling, A., Parks, G. K., Wygant, J. R., Dombecck, J., Mozer, F. S., Russell, C. T., Streltsov, A. V., and Lotko, W.: Some properties of Alvén waves: Observations in the tail lobes and the plasma sheet boundary layer, J. Geophys. Res., 110, A10S11, doi:10.1029/2004JA010 907, 2005.

Korth, A., Friedel, R. H. W., Frutos-Alfaro, F., Mouikis, C. G., and Zong, Q.: Ion composition of substorms during storm-time and non-storm-time periods, J. Atmos. Solar-Terr. Phys., 64(56), 561-566, 2002.
Lundin, R. and Hultqvist, B.: Ionospheric plasma escape by highaltitude electric fields: Magnetic moment "pumping", J. Geophys. Res., 94(A6), 6665-6680, 1989.

Lysak, R. L. and Song, Y.: Energetics of the ionospheric feedback instability, J. Geophys. Res., 107(A8), 1160, doi:10.1029/2001JA000308, 2002.

Mishin, E., Burke, W. J., Huang, C. Y., and Rich, F. J.: Electromagnetic wave structures within subauroral polarization streams, J. Geophys. Res., 108(A8), 1309, doi:10.1029/2002JA009793, 2003.

Rème, H., Bosqued, J. M., Sauvaud, J. A., Cros, A., Dandouras, J., Aoustin, C., Bouyssou, J., Camus, T., Cuvilo, J., Martz, C., Médale, J. L., Perrier, H., Romefort, D., Rouzaud, J., d'Uston, C., Möbius, E., Crocker, K., Granoff, M., Kistler, L. M., Popecki, M., Hovestadt, D., Klecker, B., Paschmann, G., Scholer, M., Carlson, C. W., Curtis, D. W., Lin, R. P., McFadden, J. P., Formisano, V., Amata, E., Bavassano-Cattaneo, M. B., Baldetti, P., Belluci, G., Bruno, R., Chionchio, G., di Lellis, A., Shelley, E. G., Ghielmetti, A. G., Lennartsson, W., Korth, A., Rosenbauer, H., Lundin, R., Olsen, S., Parks, G. K., McCarthy, M., and Balsiger, H.: The Cluster Ion Spectrometry [CIS] Experiment, Space Sci. Rev., 79, 303-350, 1997.

Streltsov, A. V. and Lotko, W.: Small-scale electric fields in downward auroral current channels, J. Geophys. Res., 108(A7), 1289, doi:10.1029/2002JA009806, 2003.

Tsurutani, B. T. and Gonzalez, W. D.: The cause of high intensity long-duration conitnuous AE activity (HILDCAAs): Interplanetary Alfvén waves trains, Planet. Space Sci., 35(4), 405-412, 1987.

Wygant, J. R., Keiling, A., Cattell, C. A., Johnson, M., Lysak, R. L., Temrin, M., Mozer, F. S., Kletsing, C. A., Scudder, J. D., Peterson, W., Russell, C. T., Parks, G., Brittnacher, M., Germany, G., and Spann, J.: Polar spacecraft based comparisons of intense electric fields and Poynting flux near and within the plasma sheet-tail lobe boundary to UVI images: An energy source for the aurora, J. Geophys. Res., 105(A8), 18 675-18 692, 2000.

Wygant, J. R., Keiling, A., Cattell, C. A., Lysak, R. L., Temerin, M., Mozer, F. S., Kletzing, C. A., Scudder, J. D., Streltsov, V., Lotko, W., and Russell, C. T.: Some properties of Alvén waves: Observations in the tail lobes and the plasma sheet boundary layer, J. Geophys. Res., 107(A8), 1201, doi:10.1029/2001JA900 113, 2002. 